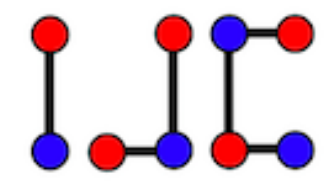

\title{
Certain topological indices and polynomials for the semitotal-point graph and line graph of semi- total-point graph for Dutch windmill graph
}

\author{
Salma Kanwal ${ }^{\mathrm{a}}$, Mariam Imtiaz ${ }^{\mathrm{b}}$, Nazeeran Idrees ${ }^{\mathrm{c}}$, Ayesha Manzoor ${ }^{\mathrm{a}}$, Ammara \\ Afzal $^{\mathrm{a}}$ \\ ${ }^{a}$ Lahore College for Women University, Lahore-Pakistan \\ ${ }^{b}$ University of Engineering and Technology, KSK Campus Lahore-Pakistan \\ ${ }^{c}$ Government College University, Faisalabad-Pakistan \\ salma.kanwal055@gmail.com, mariamimtiaz@uet.edu.pk, nazeran-jawwad@gmail.com, \\ ayeshamanzoor931@gmail.com, ammaraafzal864@yahoo.com
}

\begin{abstract}
Dutch windmill graph $[8,26]$, denoted by $D_{m}^{n}$ is a well known family of graphs with cycles. Order and size of Dutch windmill graph are $(n-1) m+1$ and $m n$ respectively. In this paper, we computed certain topological indices and polynomials i.e. Zagreb polynomials, hyper Zagreb indices, Redefined Zagreb indices, modified first Zagreb index, Reduced second Zagreb index, Reduced Reciprocal Randić index, $1^{\text {st }}$ Gourava index, $2^{\text {nd }}$ Gourava index, $1^{\text {st }}$ hyper Gourava index, $2^{\text {nd }}$ hyper Gourava index, Product connectivity Gourava index, Sum connectivity Gourava index, Forgotten index, Forgotten polynomials, M-polynomials and some topological indices in terms of M-polynomials i.e. $1^{\text {st }}$ Zagreb index , $2^{\text {nd }}$ Zagreb index, Modified $2^{\text {nd }}$ Zagreb, Randić index, Reciprocal Randić index, Symmetric division index, Harmonic index, Inverse Sum indeg index, Augmented Zagreb index for the semitotal-point graph and line graph of semitotal-point graph for Dutch windmill graph.
\end{abstract}

Keywords: Dutch windmill graph, operations on graphs, subdivision of graph, semitotal-point graph, line graph

Mathematics Subject Classification: 05C12, 05C90

DOI: $10.19184 /$ ijc.2019.3.2.1

Received: 31 May 2018, Revised: 12 Dec 2019, Accepted: 19 Dec 2019. 


\section{Definitions, Notations and Results}

All the graphs in this paper are simple, finite and undirected. In a graph $G, V(G)$ and $E(G)$ are the sets of vertices and edges respectively. Let $d_{G}(u)$ denotes the degree of a vertex $u$. Topological indices have been found to be useful in establishing relation between the structure and the properties of molecules. Topological indices mainly used in Quantitative Structure Property Relationship (QSPR) and Quantitative Structure Activity Relationships (QSAR)[5]. Some topological indices are degree based and some are distance based.

The Zagreb indices were introduced more than thirty years ago by Gutman and Trinajstić [14]. After ten years, Balaban et.al named them Zagreb group index, presented by $M_{1}$ and $M_{2}$. Later it was abbreviated to Zagreb index [17], where $M_{1}$ and $M_{2}$ represents first Zagreb index and second Zagreb index respectively. If $d_{u}$ and $d_{v}$ are the degrees of vertices $u, v$ for simple graph $G$, then first Zagreb index $[17,4]$ is defined as

$$
\begin{aligned}
& M_{1}(G)=\sum_{p \in V(G)}\left(d_{G}(p)\right)^{2} \\
& =\sum_{p q \in E(G)} d_{G}(p)+d_{G}(q)
\end{aligned}
$$

Second Zagreb index is defined as

$$
M_{2}(G)=\sum_{p q \in E(G)} d_{G}(p) d_{G}(q)
$$

Third Zagreb index introduced by Fath-Tabar [23] in 2011. Which is denoted by $Z G_{3}(G)$ for a simple graph $G$ and defined as

$$
Z G_{3}(G)=\sum_{p q \in E(G)}\left|d_{p}-d_{q}\right|
$$

Authors proposed the concept of First, Second and Third Zagreb polynomials [23], for a simple graph $G$ defined as

$$
\begin{aligned}
Z G_{1}(G, x) & =\sum_{p q \in E(G)} x^{d_{p}+d_{q}} \\
Z G_{2}(G, x) & =\sum_{p q \in E(G)} x^{d_{p} d_{q}} \\
Z G_{3}(G, x) & =\sum_{p q \in E(G)} x^{\left|d_{p}-d_{q}\right|}
\end{aligned}
$$

The Modified Zagreb index [15] is also an important degree based graph invariant. First modified Zagreb index for a graph $H$ is denoted by ${ }^{m} M_{1}(G)$, defined as

$$
\begin{array}{r}
{ }^{m} M_{1}(G)=\sum_{q \in V(G)} \frac{1}{\left(d_{q}\right)^{2}} \\
=\sum_{p q \in E(G)} \frac{1}{d_{p}+d_{q}} .
\end{array}
$$


Similarly second modified Zagreb indices is denoted by ${ }^{m} M_{2}(G)$, defined as

$$
{ }^{m} M_{2}(G)=\sum_{p q \in E(G)} \frac{1}{d_{p} d_{q}} .
$$

Shirdel et.al[27] brought into notice a new degree based graph invariant named as hyper-Zagreb index, which is defined as

$$
H M(G)=\sum_{p q \in E(G)}\left(d_{p}+d_{q}\right)^{2}
$$

Forgotten topological index is also a degree based topological index, denoted by $F(G)$ for simple graph $G$. It was presented in [10], defined as

$$
\begin{gathered}
F(G)=\sum_{q \in V(G)}\left(d_{q}\right)^{3} \\
=\sum_{p q \in E(G)}\left[\left(d_{p}\right)^{2}+\left(d_{q}\right)^{2}\right]
\end{gathered}
$$

Forgotten polynomial for a graph $G$ is defined as

$$
F(G, x)=\sum_{p q \in E(G)} x^{\left[\left(d_{p}\right)^{2}+\left(d_{q}\right)^{2}\right]}
$$

Randić connectivity index was put forward by Randić in 1975, defined as

$$
\begin{aligned}
R_{-1 / 2}(G)= & \sum_{p q \in E(G)}\left(d_{p} d_{q}\right)^{-1 / 2} \\
& =\sum_{p q \in E(G)} \frac{1}{\sqrt{d_{p} d_{q}}}
\end{aligned}
$$

and extended to general Randic connectivity index [16]. If $\alpha$ is a real number, then it is defined as

$$
R_{\alpha}(G)=\sum_{p q \in E(G)}\left(d_{p} d_{q}\right)^{\alpha}
$$

In 2014, I. Gutman et.al. [13] proposed reciprocal Randić, reduced second Zagreb and reduced reciprocal Randić index, these are degree based graph invariants, defined as

$$
\begin{gathered}
R R(G)=\sum_{p q \in E(G)} \sqrt{d_{p} d_{q}} \\
R M_{2}(G)=\sum_{p q \in E(G)}\left(d_{p}-1\right)\left(d_{q}-1\right)
\end{gathered}
$$

and

$$
R R R(G)=\sum_{p q \in E(G)} \sqrt{\left(d_{p}-1\right)\left(d_{q}-1\right)}
$$


Harmonic index for a graph $G$ defined as

$$
H(G)=\sum_{p q E(G)} \frac{2}{d_{p}+d_{q}}
$$

Many researchers are working on these graph invariants as only one index does not fully describe chemical properties of a chemical structure. Recently in 2017, V. R. Kulli proposed many new graph invariants $[18,19,20,21]$, known as family of Gourava indices, defined as

$$
\text { First Gourava Index }=G_{1} O(G)=\sum_{p q \in E(G)}\left[d_{p}+d_{q}+d_{p} d_{q}\right]
$$

and

$$
\text { Second Gourava Index }=G_{2} O(G)=\sum_{p q \in E(G)}\left[\left(d_{p}+d_{q}\right)\left(d_{p} d_{q}\right)\right]
$$

respectively.

We can rewrite second Gourava index as

$$
=\sum_{p q \in E(G)}\left[d_{p}^{2} d_{q}+d_{q}^{2} d_{p}\right]
$$

Product Connectivity Gourava index is denoted by $P G O$ and defined as

$$
P G O(G)=\sum_{p q \in E(G)} \frac{1}{\sqrt{\left(d_{p}+d_{q}\right)\left(d_{p} d_{q}\right)}}
$$

Sum connectivity Gourava index is given by

$$
S G O(G)=\sum_{p q \in E(G)} \frac{1}{\sqrt{\left(d_{p}+d_{q}\right)+\left(d_{p} d_{q}\right)}} .
$$

Hyper-Gourava indices i.e. first and second hyper Gourava index are defined as

$$
H G O_{1}(G)=\sum_{p q \in E(G)}\left[\left(d_{p}+d_{q}\right)+\left(d_{p} d_{q}\right)\right]^{2}
$$

and

$$
H G O_{2}(G)=\sum_{p q \in E(G)}\left[\left(d_{p}+d_{q}\right)\left(d_{p} d_{q}\right)\right]^{2}
$$

respectively. There are some new degree based graph invariants, which plays an important role in chemical graph theory. These topological indices are quite useful for determining total surface area and heat formation of some chemical compounds. These graph invariants are as follows Symmetric division index

$$
S D D(G)=\sum_{p q \in E(G)}\left\{\frac{\min \left(d_{p}, d_{q}\right)}{\max \left(d_{p}, d_{q}\right)}+\frac{\max \left(d_{p}, d_{q}\right)}{\min \left(d_{p}, d_{q}\right)}\right\}
$$


inverse sum index

$$
I(G)=\sum_{p q \in E(G)} \frac{d_{p} d_{q}}{d_{p}+d_{q}}
$$

and augmented Zagreb index for a graph $H$

$$
A(G)=\sum_{p q \in E(G)}\left\{\frac{d_{p} d_{q}}{d_{p}+d_{q}-2}\right\}^{3}
$$

In 2009, Zhou and Trinajstić proposed sum-connectivity index defined as

$$
\chi_{-1 / 2}(G)=\sum_{p q \in E(G)}\left[\left(d_{p}+d_{q}\right)\right]^{-1 / 2}
$$

In 2013, Ranjini [25] introduced redefined Zagreb indices i.e. redefined first, second and third Zagreb indices of a graph $G$. For a graph $G$, these indices were computed by following formulas i.e. redefined first Zagreb index,

$$
R e Z G_{1}(G)=\sum_{p q \in E(G)} \frac{d_{p}+d_{q}}{d_{p} d_{q}}
$$

redefined second Zagreb index

$$
\operatorname{Re} Z G_{2}(G)=\sum_{p q \in E(G)} \frac{d_{p} d_{q}}{d_{p}+d_{q}}
$$

and redefined third Zagreb index

$$
R e Z G_{3}(G)=\sum_{p q \in E(G)}\left(d_{p} d_{q}\right)\left(d_{p}+d_{q}\right)
$$

As algebraic polynomials play quite significant role in determining the bioactivity of chemical compounds, so some algebraic polynomials are also considered in this work. M-polynomial [1] is also one of these useful algebraic polynomials, defined as

$$
M(G, x, y)=\sum_{\delta \leq i \leq j \leq \Delta} m_{i j}(H) x^{i} y^{j}
$$

where $\delta=\min \left\{d_{G}(p)\right\}, \Delta=\max \left\{d_{G}(p)\right\}$ and $m_{i j}=|E(G)|$ for $p, q \in V(G)$ of graph $G$. Some of the topological indices are directly determined by $M-$ polynomial for $x=y=1$. These topological indices are stated as (refer to [1])

First Zagreb index

$$
M_{1}(G)=\left(D_{x}+D_{y}\right)(M(G ; x, y))_{x=y=1}
$$

Second Zagreb index

$$
M_{2}(G)=\left(D_{x} D_{y}\right)(M(G ; x, y))_{x=y=1}
$$


Certain topological indices ...

S. Kanwal, M. Imtiaz, N. Idrees, A. Manzoor and A. Afzal

Modified second Zagreb index

$$
{ }^{m} M_{2}(G)=\left(S_{x} S_{y}\right)(M(G ; x, y))_{x=y=1}
$$

Randić index

$$
R_{\alpha}(G)=\left(S_{x}^{\alpha} S_{y}^{\alpha}\right)(M(G ; x, y))_{x=y=1}
$$

Inverse Randić index

$$
R R(G)=\left(D_{x}^{\alpha} D_{y}^{\alpha}\right)(M(G ; x, y))_{x=y=1}
$$

Symmetric division index

$$
S D D(G)=\left(D_{x} S_{y}+D_{y} S_{x}\right)(M(G ; x, y))_{x=y=1}
$$

Harmonic index

$$
H(G)=2 S_{x} J(M(G ; x, y))_{x=y=1}
$$

Inverse sum index

$$
I(G)=S_{x} J D_{x} D_{y}(M(G ; x, y))_{x=y=1}
$$

and Augmented Zagreb index

$$
A(G)=S_{x}^{3} Q_{-2} D_{x}^{3} D_{y}^{3}(M(G ; x, y))_{x=y=1}
$$

Where

$$
\begin{aligned}
D_{x} M(G(x, y)) & =x \frac{\partial(M(G(x, y)))}{\partial x} \\
D_{y} M(G(x, y)) & =y \frac{\partial(M(G(x, y)))}{\partial y} \\
S_{x} M(G(x, y)) & =\int_{0}^{x} \frac{M(G(t, y))}{t} d t \\
S_{y} M(G(x, y)) & =\int_{0}^{x} \frac{M(G(x, t))}{t} d t \\
J M(G(x, y)) & =M(G(x, x))
\end{aligned}
$$

and

$$
Q_{\alpha} M(G(x, y))=x^{\alpha} M(G(x, y)) .
$$

Since last thirty years, many scholars and researchers have been working on composite graphs. There are various graph operations which are applied directly on simple graphs to study their properties under these operations. Many authors computed several topological indices of some graph operations and line graph of these graph operations for certain families $[24,11,7,6,22$, 2, 28], e.g. composition, disjunction, Cartesian product, corona product, indu-bala product and wreath product of two graphs.

Subdivision $S(G)[7,6,22,2,28]$ of a graph is acquired by embedding a vertex referred as the white vertex into each edge of $G$. Two black vertices are related in $S(G)$ if they are adjacent in 
$G$. So semitotal-point graph $R(G)$ is obtained from $S(G)$ by joining each pair of related black vertices. Line graph $L(G)$ is another graph operation, that was thoroughly considered in the precise starting work of the structural chemistry. In 1981, Bertz [3] introduced first topological index based on line graph. Many authors computed the line graph of certain families of graphs in $[12,9,24,11$, 2]. Many authors computed several topological indices for these four graph operations. M. Faisal et.al. [24] computed $A B C_{4}$ and $G A_{5}$ indices of the line graph of tadpol, wheel and ladder graphs using the notion of subdivision. In [11] Y.Gao et.al. provided the formulas for some topological indices of line graphs of the subdivision graphs of Nanotube and Nanotorus of $T U C_{4} C_{8}$. M. Reza et.al. [7] considered Wiener index and Hosoya polynomial of the line graph of the wheel graphs using the concept of subdivision and explored new results. He [6] also executed the exact values of Schultz and modified Schultz polynomial of the subdivision graph and line graph subdivision graph for wheel. M. Ajmal et.al. [2] worked on forgotten polynomial and forgotten index of line graphs of Banana tree graphs, Firecracker graph and subdivision graphs.

\section{Certain Topological Indices and polynomials of $R\left(D_{n}^{m}\right)$}

In this section, we compute Zagreb polynomials, hyper Zagreb index, Redefined Zagreb indices, modified first Zagreb index, Reduced second Zagreb index, Reduced Reciprocal Randić index, $1^{\text {st }}$ Gourava index, $2^{\text {nd }}$ Gourava index, $1^{\text {st }}$ hyper Gourava index, $2^{\text {nd }}$ hyper Gourava index, Product connectivity Gourava index, Sum connectivity Gourava index, Forgotten index, Forgotten polynomials, M-polynomials and some topological indices in terms of M-polynomials i.e. $1^{\text {st }} \mathrm{Za}$ greb index, $2^{\text {nd }}$ Zagreb index, Modified $2^{\text {nd }}$ Zagreb index, Randić index, Reciprocal Randić index, Symmetric division index, Harmonic index, Inverse Sum indeg index, Augmented Zagreb index for the semitotal-point graph of Dutch windmill graph i.e. $R\left(D_{n}^{m}\right)$. As the semitotal-point graph of Dutch windmill graph is shown in figure 2 .
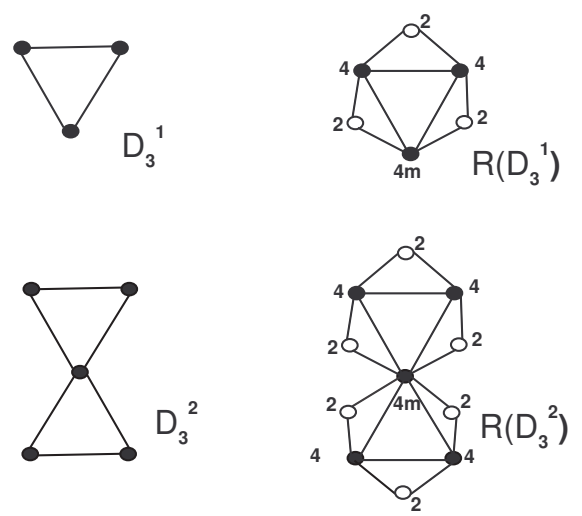

Figure 1. Semitotal-point graph of Dutch windmill graph, i.e. $R\left(D_{n}^{m}\right)$

Theorem 2.1. Let $G$ be the Semitotal-point graph of Dutch windmill graph, i.e. $R\left(D_{n}^{m}\right)$. Then the forgotten polynomial and Zagreb polynomials are given by 


$$
\begin{aligned}
F(G, x) & =2 m\left(x^{16 m^{2}+4}+x^{16 m^{2}+16}\right)+(m n-2 m) x^{2}+(2 m n-2 m) x^{20} \\
Z G_{1}(G, x) & =2 m x^{4 m} x^{2}\left(x^{2}+1\right)+m n x^{6}\left(x^{2}+2\right)-2 m x^{6}\left(x^{2}+1\right) \\
Z G_{2}(G, x) & =2 m x^{8 m}\left(1+x^{8 m}\right)+m n\left(2+x^{8}\right)-2 m n x^{8}\left(1+x^{8}\right) \\
Z G_{3}(G, x) & =2 m\left(x^{4 m-2}+x^{4 m-4}\right)+m n\left(1+2 x^{2}\right)-2 m\left(1+x^{2}\right)
\end{aligned}
$$

Proof. Consider the Semitotal-point graph of Dutch windmill graph, denoted by $R\left(D_{n}^{m}\right)$. There are $2 m n-(m-1)$ total number of vertices and $3 m n$ total number of edges in $R\left(D_{n}^{m}\right) . R\left(D_{n}^{m}\right)$ contains the vertices with degrees $4 m, 4$ and 2. Degree based edge partition of $R\left(D_{n}^{m}\right)$ is denoted by $E_{\left(d_{p}, d_{q}\right)}$, where $p q \in E\left(R\left(D_{n}^{m}\right)\right)$. The edge partition $E_{(4 m, 2)}$ contains $2 m$ edges for $d_{p}=4 m$ and $d_{q}=2$, the edge partition $E_{(4 m, 4)}$ contains $2 m$ edges for $d_{p}=4 m$ and $d_{q}=4$, edge partition $E_{(4,4)}$ contains $(n-2) m$ edges for $d_{p}=4$ and $d_{q}=4$ and the edge partition $E_{(2,4)}$ contains $(2 n-2) m$ edges for $d_{p}=2$ and $d_{q}=4$, shown in Table 1

Table 1. Edge partition of $R\left(D_{n}^{m}\right)$

\begin{tabular}{ll}
\hline Edges of type & $\begin{array}{l}\text { Number of } \\
\text { edges }\end{array}$ \\
\hline$E_{(4 m, 2)}$ & $2 m$ \\
$E_{(4 m, 4)}$ & $2 m$ \\
$E_{(4,4)}$ & $(n-2) m$ \\
$E_{(2,4)}$ & $(2 n-2) m$ \\
\hline
\end{tabular}

\section{Forgotten polynomial :}

$$
\begin{aligned}
\mathbf{F}(\mathbf{G}, \mathbf{x}) & =\sum_{\mathbf{p} \mathbf{q} \in \mathbf{E}(\mathbf{G})} \mathbf{x}^{\left(\mathbf{d}_{\mathbf{p}}\right)^{\mathbf{2}}+\left(\mathbf{d}_{\mathbf{q}}\right)^{\mathbf{2}}} \\
& =2 m\left(x^{16 m^{2}+4}+x^{16 m^{2}+16}\right)+(m n-2 m) x^{2}+(2 m n-2 m) x^{20}
\end{aligned}
$$

\section{$1^{\text {st }}$ Zagreb polynomial :}

$$
\begin{aligned}
\mathbf{Z G}_{\mathbf{1}}(\mathbf{G}, \mathbf{x}) & =\sum_{\mathbf{p} \mathbf{q} \in \mathbf{E}(\mathbf{G})} \mathbf{x}^{\mathbf{d}_{\mathbf{p}}+\mathbf{d}_{\mathbf{q}}} \\
& =\sum x^{4 m+2}+\sum x^{4 m+4}+\sum x^{4+4}+\sum x^{2+4} \\
& =2 m\left(x^{4 m+2}\right)+2 m x^{4 m+4}+(n m-2 m) x^{4+4}+(2 n m-2 m) x^{2+4} \\
& =2 m x^{4 m} x^{2}+2 m x^{4 m} x^{4}+m n x^{8}-2 m x^{8}+2 m n x^{6}-2 m x^{6} \\
& =2 m x^{4 m} x^{2}\left(x^{2}+1\right)+m n x^{6}\left(x^{2}+2\right)-2 m x^{6}\left(x^{2}+1\right)
\end{aligned}
$$


$2^{\text {nd }}$ Zagreb polynomial:

$$
\begin{aligned}
\mathbf{Z G}_{\mathbf{2}}(\mathbf{G}, \mathbf{x}) & =\sum_{\mathbf{p} \mathbf{q} \in \mathbf{E}(\mathbf{G})} \mathbf{x}^{\mathbf{d}_{\mathbf{p}} \mathbf{d}_{\mathbf{q}}} \\
& =\sum x^{4 m \times 2}+\sum x^{4 m \times 4}+\sum x^{4 \times 4}+\sum x^{2 \times 4} \\
& =2 m x^{8 m}+2 m x^{16 m}+m n x^{16}-2 m x^{16}+2 m n x^{8}-2 m x^{8} \\
& =2 m x^{8 m}\left(1+x^{8 m}\right)+m n x^{8}\left(2+x^{8}\right)-2 m x^{8}\left(1+x^{8}\right) \\
& =2 m x^{8}\left(1+x^{8}\right)+\left(1+x^{8}\right) x^{8} m[n-2] \\
& =2 m x^{4}\left(x^{m}+n-1\right)
\end{aligned}
$$

$3^{r d}$ Zagreb polynomial:

$$
\begin{aligned}
& \quad \mathbf{Z G}_{\mathbf{3}}(\mathbf{G}, \mathbf{x})=\sum_{\mathbf{p q} \in \mathbf{E}(\mathbf{G})} \mathbf{x}^{\left|\mathbf{d}_{\mathbf{p}}-\mathbf{d}_{\mathbf{q}}\right|} \\
& =\sum x^{4 m-2}+\sum x^{4 m-4}+\sum x^{4-4}+\sum x^{2-4} \\
& =2 m x^{4 m-2}+2 m x^{4 m-4}+m n x^{0}-2 m x^{0}+2 m n x^{2}-2 m x^{2} \\
& =2 m\left(x^{4 m-2}+x^{4 m-4}\right)+m n-2 m+2 m n x^{2}-2 m x^{2} \\
& =2 m\left(x^{4 m-2}+x^{4 m-4}\right)+m n\left(1+2 x^{2}\right)-2 m\left(1+x^{2}\right)
\end{aligned}
$$

Theorem 2.2. Let $G$ be the semitotal-point graph of a Dutch Windmill graph. Then

$$
\begin{aligned}
H M(G) & =64 m^{3}+96 m^{2}-160 m+136 m n \\
\operatorname{Re} Z G_{1}(G) & =1-m+2 m n \\
\operatorname{Re} Z G_{2}(G) & =\frac{16 m^{2}}{4 m+2}+\frac{32 m}{4 m+4}+\frac{14}{3} m n-\frac{20}{3} m \\
\operatorname{ReZ} G_{3}(G) & =192 m^{3}+160 m^{2}-352 m+224 m n \\
{ }^{m} M_{2}(G) & =m\left[\frac{1}{2 m+1}+\frac{1}{2 m+2}-\frac{7}{12}\right]+m n \frac{11}{24} \\
R M_{2}(G) & =32 m^{2}-32 m+15 m n \\
R R R(G) & =2 m \sqrt{4 m-1}+2 m \sqrt{12 m-3}+3 m n-6 m+2 m n \sqrt{(3)}-2 m \sqrt{(3)} \\
G O_{1}(G) & =64 m^{2}-64 m+52 m n \\
G O_{2}(G) & =192 m^{3}+160 m^{2}-352 m+224 m n \\
H G O_{1} & =1088 m^{3}+416 m^{2}-1504 m+968 m n \\
H G O_{2} & =10240 m^{5}+18432 m^{4}+8704 m^{3}-37376 m+20992 m n \\
P G O(G) & =m \frac{1}{\sqrt{8 m^{2}+4 m}}+m \frac{1}{4 \sqrt{m^{2}+m}}+m n \frac{1}{8 \sqrt{2}}-m \frac{1}{4 \sqrt{2}}+m n \frac{1}{2 \sqrt{3}}-m \frac{1}{2 \sqrt{3}}
\end{aligned}
$$


Proof. Apply Formulas (5), (19), (20), (21), (25), (9), (10), (11),(13), (16), (17), (1), (15) and (6) to the edge partitions shown in Table 1 to get the required results.

Theorem 2.3. Let $G$ be the semitotal-point graph of a Dutch Windmill graph. Then M-polynomial and certain topological indices in term of M-polynomial are

$$
\begin{aligned}
M(G ; x, y) & =2 m x^{4 m} y^{2}\left(1+y^{2}\right)+m x^{4} y^{4}(n-2)+2 m x^{2} y^{4}(n-1) \\
M_{1}(G) & =16 m^{2}+20 m n-16 m \\
M_{2}(G) & =48 m^{2}+32 m n-48 m \\
m^{m} M_{2}(G) & =\frac{3}{8}+\frac{5 m n}{16}-\frac{3 m}{8} \\
R_{\alpha}(G) & =\frac{2 m}{(8 m)^{\alpha}}+\frac{2 m}{(16 m)^{\alpha}}+\frac{m(n-2)}{\left(16^{\alpha}\right)}+\frac{2 m(n-1)}{\left(8^{\alpha}\right)} \\
R R_{\alpha}(G) & =2 m(8 m)^{\alpha}+2 m(16 m)^{\alpha}+\left(16^{\alpha}\right)(n-2)+(2 n-2) m\left(8^{\alpha}\right) \\
S D D(G) & =6 m^{2}+7 m n-9 m+3 \\
H(G) & =\frac{4 m}{4 m+2}+\frac{4 m}{4 m+4}-\frac{7 m}{12}+\frac{11 m}{24} \\
I(G) & =\frac{16 m^{2}}{4 m+2}+\frac{32 m^{2}}{4 m+4}+\frac{16 m n}{8}-\frac{32 m}{8}+\frac{16 m n}{6}-\frac{16 m}{6} \\
A(G) & =16 m+\frac{8192 m^{4}}{(4 m+2)^{3}}+\frac{4096(n-2)}{216}+\frac{1024 m(n-1)}{64}
\end{aligned}
$$

Proof. Apply Formulas (22), (23), (24), (25), (26), (27), (28), (29), (30) and (31) to the edge partitions shown in the Table 1 to get the required results.

\section{Certain Topological Indices and polynomials of $L\left(R\left(D_{n}^{m}\right)\right)$}

In this section, we compute Zagreb polynomials, hyper Zagreb, Redefined Zagreb indices, modified first Zagreb, Reduced second Zagreb, Reduced Reciprocal Randić, 1st Gourava index, 2nd Gourava index, 1st hyper Gourava index, 2nd hyper Gourava index, Product connectivity Gourava index, Sum connectivity Gourava index, Forgotten index, Forgotten polynomials, Mpolynomials and some topological indices in term of the M-polynomials i.e 1st zagreb index, 2nd Zagreb index, Modified 2nd Zagreb, Randić index, Reciprocal Randić index, Symmetric division, Harmonic index, Inverse Sum index, Augmented Zagreb index for the line graph of semitotal point graph of Dutch windmill graph. As the line graph of semitotal point graph of Dutch windmill graph is shown in figure 3 .

Theorem 3.1. Let $G$ be the Line graph of semitotal point graph of a Dutch windmill graph i.e $L\left(R\left(D_{n}^{m}\right)\right)$. Then forgotten polynomial and Zagreb polynomials are given by

$$
\begin{aligned}
F(G, x) & =m^{2}\left[2 x^{32 m^{2}+32 m+8}+4 x^{32 m^{2}+5}+2 x^{32 m^{2}}\right]+m\left[x^{16 m^{2}+16 m+20}-x^{32 m^{2}+32 m+8}\right. \\
& \left.+2 x^{16 m^{2}+16 m+40}-x^{32 m^{2}}+2 x^{16 m^{2}+16}-3 x^{72}-3 x^{32}-3 x^{52}\right]+m n\left[x^{72}+2 x^{32}+4 x^{52}\right] \\
Z G_{1}(G, x) & =m^{2}\left[2 x^{8 m+4}+4 x^{8 m+4}+2 x^{8 m}\right]+m\left[4 x^{4 m+6}-x^{8 m+4}+2 x^{4 m+8}-x^{8 m}+2 x^{4 m+4}\right. \\
& \left.-3 x^{12}-3 x^{8}-8 x^{10}\right]+m n\left[2 x^{8}+4 x^{10}+x^{12}\right] \\
Z G_{2}(G, x) & =m^{2}\left[2 x^{16 m^{2}+16 m+4}+4 x^{16 m^{2}+16 m+4}+3 x^{16 m^{2}}\right]+m\left[4 x^{64 m+32}-x^{16 m^{2}+16 m+4}\right. \\
& \left.+2 x^{24 m+12}-x^{16 m^{2}}+2 x^{16 m}-3 x^{36}-3 x^{16}\right]+m n\left[x^{16}+x^{36}+4 x^{24}\right] \\
Z G_{3}(G, x) & =4 m^{2}\left(1+x^{2}\right)+m\left[4 x^{4 m-4}+2 x^{4 m-2}+2 x^{4 m-4}-8 x^{2}-8\right]+m n\left[3+4 x^{2}\right]
\end{aligned}
$$


Proof. Consider the Line graph of semitotal-point graph of Dutch windmill graph, denoted by $L\left(R\left(D_{n}^{m}\right)\right)$. The total no of vertices and edges in $L\left(R\left(D_{n}^{m}\right)\right)$ are $3 m n$ and $8 m^{2}+7 m n-8 m$. $R\left(D_{n}^{m}\right)$ contains the vertices with degrees $4 m+2,4 m, 4$, and 6 . We partition the edges of $L\left(R\left(D_{n}^{m}\right)\right)$ based on the edges of type $E_{\left(d_{u}, d_{v}\right)}$, where $\left.u v \in E\left(R\left(D_{n}^{m}\right)\right)\right)$. The edge partition $E_{(4 m+2,4 m+2)}$ contains $m(2 m-1)$ edges for $d_{u}=4 m+2$ and $d_{v}=4 m+2$, the edge partition $E_{(4 m+2,4 m)}$ contains $4 m^{2}$ edges for $d_{u}=4 m+2$ and $d_{v}=4 m$, the edge partition $E_{(4 m+2,4)}$ contains $4 m$ edges for $d_{u}=4 m+2$ and $d_{v}=4$, the edge partition $E_{(4 m+2,6)}$ contains $2 m$ edges for $d_{u}=4 m+2$ and $d_{v}=6$, the edge partition $E_{(4 m, 4 m)}$ contains $m(2 m-1)$ edges for $d_{u}=4 m$ and $d_{v}=4 m$, the edge partition $E_{(4 m, 4)}$ contains $2 m$ edges for $d_{u}=4 m$ and $d_{v}=4$, the edge partition $E_{(6,6)}$ contains $(n-3) m$ edges for $d_{u}=6$ and $d_{v}=6$, the edge partition $E_{(4,4)}$ contains $(2 n-3) m$ edges for $d_{u}=4$ and $d_{v}=4$, the edge partition $E_{(6,4)}$ contains $(4 n-8) m$ edges for $d_{u}=6$ and $d_{v}=4$ shown in Table 2

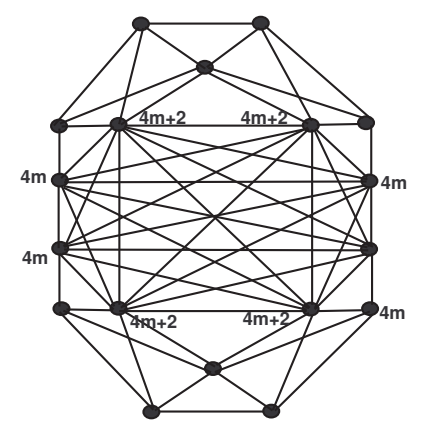

Figure 2. Line graph of Semitotal-point of Dutch windmill graph i.e. $L\left(R\left(D_{3}^{2}\right)\right)$

Table 2. Edge partition of $L\left(R\left(D_{n}^{m}\right)\right)$

\begin{tabular}{ll}
\hline Edges of type & $\begin{array}{l}\text { Number of } \\
\text { edges }\end{array}$ \\
\hline$E_{(4 m+2,4 m+2)}$ & $m(2 m-1)$ \\
$E_{(4 m+2,4 m)}$ & $4 m^{2}$ \\
$E_{(4 m+2,4)}$ & $4 m$ \\
$E_{(4 m+2,6)}$ & $2 m$ \\
$E_{(4 m, 4 m)}$ & $m(2 m-1)$ \\
$E_{(4 m, 4)}$ & $2 m$ \\
$E_{(6,6)}$ & $(n-3) m$ \\
$E_{(4,4)}$ & $(2 n-3) m$ \\
$E_{(6,4)}$ & $(4 n-8) m$ \\
\hline
\end{tabular}




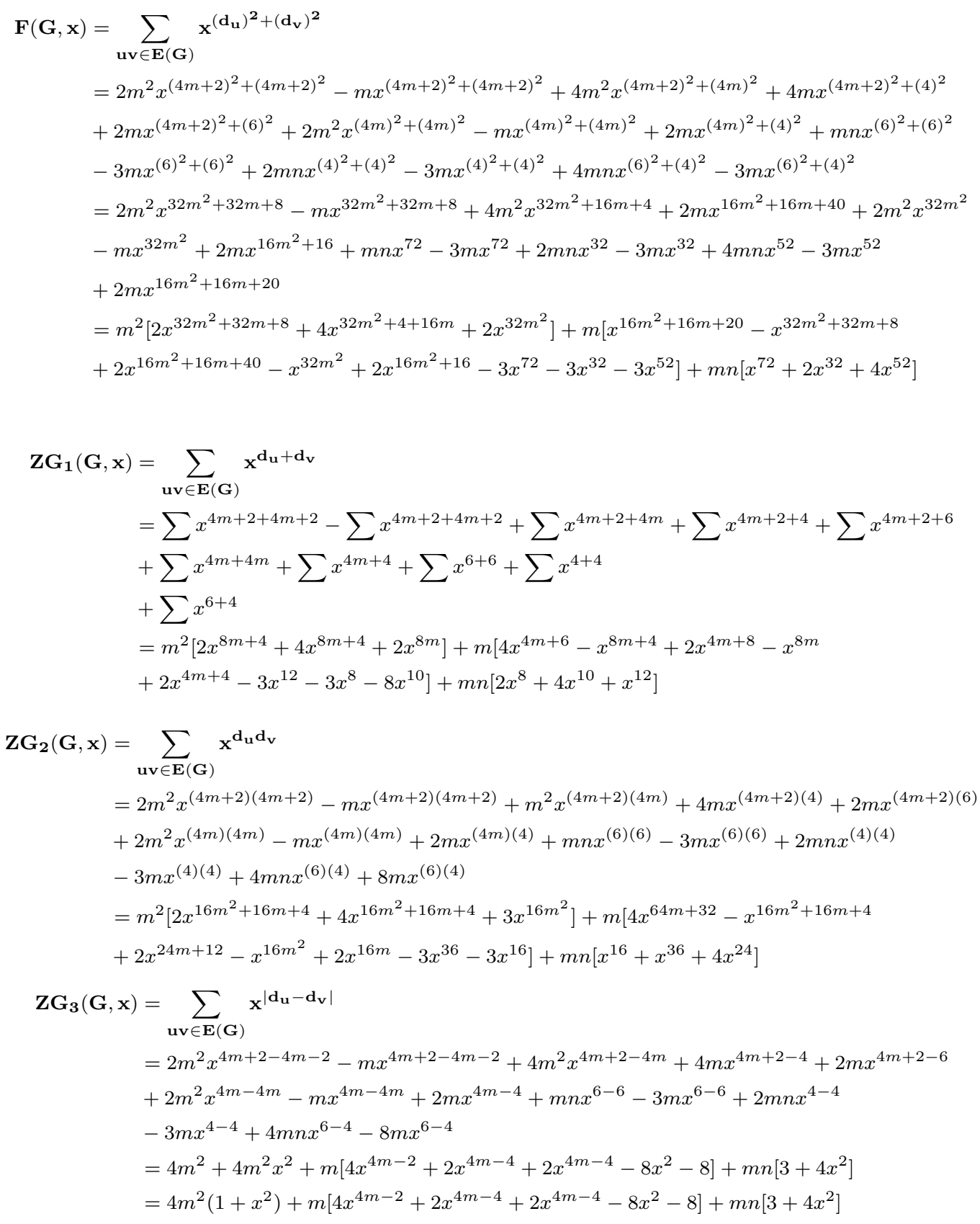


Theorem 3.2. Let $G$ be the Line graph of semitotal point graph of a Dutch Windmill graph. Then

$$
\begin{aligned}
& H M(G)=384 m^{4}+32 m^{3}+48 m^{2}-1280 m+762 m n \\
& \operatorname{Re} Z G_{1}(G)=\frac{\left(2 m^{2}-m\right)(2 m+1)}{4 m^{2}+4 m+1}+\frac{\left(4 m^{3}+m^{2}\right)}{2 m^{2}+m}+\frac{\left(2 m^{2}+3 m\right)}{(2 m+1)}+\frac{\left(2 m^{2}+4 m\right)}{(6 m+3)} \\
& +\frac{\left(2 m^{2}-m\right)}{2 m}+\frac{(m+1)}{2}+\frac{(n-3) m}{3}+\frac{(2 n-3) m}{2}+\frac{(n-2) 5 m}{3} \\
& \operatorname{Re} Z G_{2}(G)=8 m^{4}+m^{3}\left[\frac{8}{2 m+1}-\frac{4}{m+2}+\frac{32}{4 m+1}+4\right]+m^{2}\left[\frac{4}{4 m+2}-\frac{4}{2 m+1}+\frac{8}{m+1}\right. \\
& \left.+\frac{16}{4 m+1}+\frac{16}{2 m+3}+\frac{12}{m+2}-2\right]+m\left[-15-\frac{1}{m+1}+\frac{16}{2 m+3}+\frac{6 m}{m+2}\right]+\frac{83 m n}{5} \\
& \operatorname{Re} Z G_{3}(G)=768 m^{5}+512 m^{4}+512 m^{3}+928 m^{2}-3424 m+1648 m n \\
& { }^{m} M_{2}(G)=m^{2}\left[\frac{1}{2 m+1}+\frac{2}{4 m+1}\right]+m\left[\frac{2}{2 m+3}-\frac{1}{8 m+4}+\frac{1}{2 m+4}+\frac{1}{2 m+2}-\frac{47}{40}\right] \\
& +\frac{13 m n}{20}-\frac{1}{8} \\
& R M_{2}(G)=128 m^{4}-32 m^{3}+116 m^{2}-208 m+103 m n \\
& R R R(G)=m^{2}\left[2 \sqrt{16 m^{2}+8 m+1}+4 \sqrt{16 m^{2}-1}+2 \sqrt{16 m^{2}-8 m+1}-\sqrt{16 m^{2}+8+1}\right] \\
& +m\left[4 \sqrt{12 m+3}+2 \sqrt{20 m+5}-\sqrt{16 m^{2}-8 m+1}+2 \sqrt{12 m-3}-8 \sqrt{5}-24\right] \\
& +m n[4 \sqrt{15}+11] \\
& G O_{1}(G)=128 m^{4}+96 m^{3}+168 m^{2}-408 m+232 m n \\
& G O_{2}(G)=768 m^{5}+512 m^{4}+512 m^{3}+928 m^{2}-3424 m+1648 m n \\
& H G O_{1}=1536 m^{6}+3584 m^{5}+2048 m^{4}+4096 m^{3}+4560 m^{2}-16338 m+8080 m n \\
& H_{G O} O_{2}=449792 m n-1069624 m+32768 m^{8}-16384 m^{6}+128 m^{3}+128 m^{2} \\
& +\left(256 m^{4}+192 m^{3}+32 m^{2}\right)^{2}+m\left(128 m^{2}+256 m+96\right)^{2}+2 m\left(96 m^{2}+240 m+96\right)^{2} \\
& +\left(2 m^{2}-m\right)\left(128 m^{3}+192 m^{2}+96 m+16\right)^{2} \\
& P G O(G)=\frac{m^{2}}{64 m^{3}+96 m^{2}+48 m+8}+\frac{m}{16 m^{2}+32 m+12}+\frac{m}{32 m^{2}+24 m+4} \\
& +\frac{m}{16 m^{2}+32 m+12}+\frac{m}{48 m^{2}+122 m+48}+\frac{1}{64 m}-\frac{1}{128 m^{2}}+\frac{1}{32 m+32} \\
& +\frac{4 m n}{432}-\frac{m}{144}+\frac{m n}{60}-\frac{m}{30}+\frac{12 m n}{3072}-\frac{8 m}{3072} \\
& S G O(G)=\frac{m^{2}}{8 m^{2}+12 m+4}-\frac{m}{16 m^{2}+24 m+8}+\frac{2 m^{2}}{8 m^{2}+8 m+1}+\frac{2 m}{10 m+7}+\frac{m}{14 m+10} \\
& +\frac{m}{4+8 m}-\frac{1}{8+16 m}+\frac{m}{10 m+2}+\frac{m n}{48}-\frac{m}{16}+\frac{m n}{12}-\frac{m}{8}+\frac{m n}{6}-\frac{m}{3072} \\
& F(G)=176 m^{4}+208 m^{3}+112 m^{2}-672 m+344 m n
\end{aligned}
$$

Proof. Apply Formulas (5), (19), (20), (21), (25), (9), (10), (11),(13), (16), (17), (1), (15) and (6) to the edge partitions shown in Table 2 to get the required results.

Theorem 3.3. Let $G$ be the Line graph of Semitotal-point graph of a Dutch Windmill graph i.e 
$L\left(R\left(D_{n}^{m}\right)\right)$. Then M-polynomial and certain topological indices in terms of M-polynomial are

$$
\begin{aligned}
& M(G ; x, y)=2 m^{2} x^{4 m+2} y^{4 m+2}-m x^{4 m+2} y^{4 m+2}+4 m^{2} x^{4 m+2} y^{4 m}+4 m x^{4 m+2} y^{4}+2 m x^{4 m+2} y^{6} \\
& +2 m^{2} x^{4 m} y^{4 m}-m x^{4 m} y^{4 m}+2 m x^{4 m} y^{4}+m n x^{6} y^{6}-3 m x^{6} y^{6}+2 m n x^{4} y^{4} \\
& -3 m x^{4} y^{4}+4 m n x^{6} y^{4}-8 m x^{6} y^{4} \\
& M_{1}(G)=64 m^{3}+32 m^{2}-96 m+68 m n \\
& M_{2}(G)=128 m^{4}+32 m^{3}+136 m^{2}-296 m+164 m n \\
& { }^{m} M_{2}(G)=\frac{m^{2}}{(2 m+1)(4 m+2)}-\frac{m}{(4 m+2)^{2}}+\frac{2 m}{4 m+2}+\frac{m}{(12 m+6)}+\frac{m}{(8 m)}-\frac{1}{(16 m)} \\
& +\frac{m}{(8 m)}+\frac{m n}{(36)}+\frac{m}{(12)}+\frac{m n}{(8)}-\frac{3 m}{(16)}+\frac{m n}{6}-\frac{m}{3} \\
& R_{\alpha}(G)=\frac{2 m^{2}}{(4 m+2)^{\alpha}(4 m+2)^{\alpha}}-\frac{m}{(4 m+2)^{\alpha}(4 m+2)^{\alpha}}+\frac{4 m^{2}}{(4 m)^{\alpha}(4 m+2)^{\alpha}}+\frac{4 m}{(4)^{\alpha}(4 m+2)^{\alpha}} \\
& +\frac{2 m}{(6)^{\alpha}(4 m+2)^{\alpha}}+\frac{2 m^{2}}{(4 m)^{\alpha}(4 m)^{\alpha}}-\frac{m}{(4 m)^{\alpha}(4 m)^{\alpha}}+\frac{2 m}{(4)^{\alpha}(4 m)^{\alpha}}+\frac{m n}{(6)^{\alpha}(6)^{\alpha}} \\
& -\frac{3 m}{(6)^{\alpha}(6)^{\alpha}}+\frac{2 m n}{(4)^{\alpha}(4)^{\alpha}}-\frac{3 m}{(4)^{\alpha}(4)^{\alpha}}+\frac{4 m n}{(4)^{\alpha}(4)^{\alpha}}-\frac{8 m}{(4)^{\alpha}(6)^{\alpha}} \\
& R R_{\alpha}(G)=2 m^{2}(4 m+2)^{\alpha}(4 m+2)^{\alpha}-m(4 m+2)^{\alpha}(4 m+2)^{\alpha}+4 m^{2}(4 m+2)^{\alpha}(4 m)^{\alpha} \\
& +4 m(4 m+2)^{\alpha}(4)^{\alpha}+2 m(4 m+2)^{\alpha}(6)^{\alpha}+2 m^{2}(4 m)^{\alpha}(4 m)^{\alpha} \\
& -m(4 m)^{\alpha}(4 m)^{\alpha}+2 m(4 m)^{\alpha}(4)^{\alpha}+m m(6)^{\alpha}(6)^{\alpha}-3 m(6)^{\alpha}(6)^{\alpha} \\
& +2 m n(4)^{\alpha}(4)^{\alpha}-3 m(4)^{\alpha}(4)^{\alpha}+4 m n(6)^{\alpha}(4)^{\alpha}-8 m(6)^{\alpha}(4)^{\alpha} \\
& S D D(G)=\left(4 m^{2}-2 m\right)+4 m^{2}\left(\frac{32 m^{2}+16 m+4}{16 m^{2}+8 m}\right)+4 m\left(\frac{16 m^{2}+16 m+20}{16 m+8}\right) \\
& +2 m\left(\frac{16 m^{2}+16 m+40}{24 m+12}\right)+\left(6 m^{2}-14 m+6 m n+2\right)+\frac{26 m n-52 m}{3} \\
& H(G)=\frac{m^{2}}{2 m+1}+\frac{4 m^{2}}{4 m+1}+m\left[\frac{2}{2 m+3}+\frac{1}{2 m+4}-\frac{1}{8 m+4}+\frac{1}{2 m+2}-\frac{3}{8}-\frac{4}{5}\right] \\
& +m n\left[\frac{1}{12}+\frac{1}{4}+\frac{2}{5}\right]-\frac{1}{8} \\
& I(G)=m^{4}\left[\frac{24}{2 m+1}\right]+m^{3}\left[\frac{12}{2 m+1}+\frac{8}{4 m+1}\right]+m^{2}\left[\frac{16}{2 m+3}-\frac{2}{4 m+1}\right] \\
& +m\left[\frac{-4}{8 m+4}-\frac{231}{5}\right]+m n\left[\frac{166}{3}\right] \\
& A(G)=\frac{\left(2 m^{2}-m\right)(4 m+2)^{6}}{(8 m+2)^{3}}+\frac{4 m^{2}\left(16 m^{2}+8 m\right)^{3}}{(8 m)^{3}} \\
& +\frac{4 m(16 m+6)^{3}}{(4 m+4)^{3}}+\frac{2 m(24 m+12)^{3}}{(4 m+6)^{3}}+\frac{\left(2 m^{2}-m\right)\left(16 m^{2}\right)^{3}}{(8 m-2)^{3}}+\frac{2 m(16 m)^{3}}{(4 m+2)^{3}} \\
& +\frac{(m n-3 m)(36)^{3}}{(10)^{3}}+\frac{(2 m n-3 m)(16)^{3}}{(6)^{3}}+\frac{(4 m n-8 m)(24)^{3}}{(8)^{3}}
\end{aligned}
$$

Proof. Apply Formulas (22), (23), (24), (25), (26), (27), (28), (29), (30) and (31) to the edge partitions shown in the Table 2 to get the required results.

\section{References}

[1] M. S. Ahmad, W. Nazeer, S. M. Kang, and C. Y. Jung, $M$-polynomials and degree based topological indices for the line graph of Firecracker graph, Glob. J. Pure Appl. Math. 13 (6) (2017), 2749-2776.

[2] M. Ajmal, W. Nazeer, W. Khalid, and S. M. Kang, Forgotten polynomial and forgotten index 
for the line graphs of Banana tree graph, Firecracker graph and subdivision graphs, Glob. J. Pure Appl. Math. 13 (6) (2017), 2673-2682.

[3] S. H. Bertz, The bond graph, J. C. S. Chem. Commun. (1981), 818-820.

[4] H. Deng, D. Sarala, S. K. Ayyaswamy, and S. Balachandran, The Zagreb index of four operations on graphs, Appl. Math. Comput. 275 (2016), 422-431.

[5] J. Devillers and A. T. Balaban (Eds.), Topological indices and related descriptors in QSAR and QSPR, Gordon and Breach, Amsterdam, The Netherlands (1999).

[6] M. R. Farahani and M. K. Jamil, The schultz and modified schultz polynomials of the certain subdivision and line subdivision graphs, J. Chem. Pharm. Res. 8 (3) (2016), 51-57.

[7] M. R. Farahani, M. K. Jamil, M. R. Rajesh Kanna, and S. M. Hosamani, The wiener index and hosaya polynomial of the Subdivision Graph of wheel $S\left(W_{n}\right)$ and the line graph subdivision of the wheel $L\left(S\left(W_{n}\right)\right)$, Appl. Math. 6 (2) (2016), 21-24.

[8] M. R. Farahani, M. K. Jamil, S. Wang, W. Gao, and B. Wei, The hosoya, schultz and modified schultz polynomials of a class of Dutch windmill graph $D_{n}^{(m)}$, Commun. Appl. Anal. 22 (1) (2017), 43-62.

[9] M. R. Farahani, M. F. Nadeem, S. Zafar, and M. N. Husin, Study of the topological indices of the line graphs of H-pantacenic nanotubes, New FRONT. Chem. 26 (1) (2017), 31-38.

[10] B. Furtula and I. Gutman, A forgotten topological index, J. Math. Chem. 53 (2015), 11841190.

[11] Y. Gao, M. K. Jamil, A. Aslam, and M. R. Farahani, Comutation of some new/old vertexdegree-based topological indices of line graph of subdivision graph of some nanosrtuctures, J. Optoelectron. Biomed. Mater. 9 (3) (2017), 135-142.

[12] I. Gutman and E. Estrada, Topological indices based on the line graph of molecular graph, $J$. Chem. Inf. Comput. Sci. 36 (1996), 541.

[13] I. Gutman, B. Furtula, and C. Elphick, Three new/old vertex-degree based topological indices, MATCH Commun. Math. Comput. Chem. 72 (24) (2014), 617-632.

[14] I. Gutman, N. Trinajstić, and C. F. Wilcox, Graph theory and molecular orbits. Total $\pi$ electron energy of alternate hydrocarbons, Chem. Phys. Lett. 17 (1972), 535-538.

[15] J. Hao, Theorems about Zagreb indices and modified Zagreb indices, MATCH Commun. Math. Comput. Chem. 65 (2011), 659-670.

[16] M. Imran and S. Akhter, The sharp bounds on general sum-connectivity index of four operations on graphs, J. Inequal. Appl. 241 (2016). 
[17] M. H. Khalifeh, H. Yousefi-Azari, and A. R. Ashrafi, The first and second Zagreb indices of some graph operations, Discrete Appl. Math. 157 (2009), 804-811.

[18] V. R. Kulli, The gourava indices and coindices of graphs, Ann. Pure Appl. Math. 14 (1) (2017), 33-38.

[19] V. R. Kulli, The product connectivity gourava index, J. Comput. Math. Sci. 8 (6) (2017), 235-242

[20] V. R. Kulli, On the sum connectivity Gourava index, Int. J. Math. Arch. 8 (6) (2017), 211-217.

[21] V. R. Kulli, On hyper-gourava indices and coindices, Int. J. Math. Arch. 8 (12) (2017), 116120.

[22] G. Mahanappriya and D. Vijayalakshmi, Topological indices of the total graph of subdivision graph, Ann. Pure Appl. Math. 14 (2) (2017), 231-235.

[23] A. E. Nabeel, Zagreb polynomials of certain families of dendrimer nanostars, Tikrit J. Pure Sci. 20 (4) (2015).

[24] M. F. Nadeem, S. Zafar, and Z. Zahid, On the certain topological indices of the line graph of subdivision graphs, Appl. Math. Comput. 217 (2015), 790-794.

[25] P. S. Ranjini, V. Lokesha, and A. Usha, Relation between phenylene and hexagonal squeeze using harmonic index. Int. J. Graph Theory 1 (2013), 116-21.

[26] M. R. Rajesh Kanna, R. P. Kumar, and R. Jagadeesh, Computation of topological indices of Dutch windmill graph, Open J. Discrete Math. 6 (2016), 74-81.

[27] G. H. Shirdel, H. Rezapour, and A. M. Syadi, The hyper-Zagreb index of graph operations, Iranian J. Math. Chem. 4 (2) (2013), 213-220.

[28] G. Srinivasa and K. Asha, Atom-bond connectivity index of subdivision graphs of some special graphs, Int. J. Adv. Res. Comput. Sci. 8 (6) (2017). 